\title{
Stimulus intensity dynamism during two-valued auditory discrimination acquisition: a systematic replication
}

SCOT BLUE ${ }^{1}$

BROWN UNIVERSITY

Using two counterpart pairs of groups, rats were trained to discriminate between $4 \mathrm{kHz}$ tones spaced $5 \mathrm{~dB}$ apart at the high or low ends of an intensity continuum. The major findings agree with those of Sadowsky (1966) who, using $10 \mathrm{~dB}$ separations, found a large amount of dynamism at high intensities, but none at low intensities. However, some details of the present results differ and may be related to the $S^{D-S \Delta}$ separation variable.

In many experimental situations where responding is measured in the presence of more than one value of an intensive stimulus, a relationship is found between the strength of the response (amplitude, force, rate, etc.) and the intensity of the stimulus. A monotonic increasing response strength-stimulus intensity relationship was called Stimulus Intensity Dynamism by Hull (1949). Recently, Gray (1965) reviewed some of the literature which demonstrates the existence of this effect and tried to categorize the situations under which it can and cannot be measured. However, until the study by Sadowsky (1966), little parametric investigation of the stimulus intensity variable had been done.

In the Sadowsky study, four counterpart pairs of groups were run. A counterpart pair consists of groups that are trained on the same stimuli, but the reinforced intensity $\left(S^{D}\right)$ for one group is the nonreinforced intensity $\left(S^{\Delta}\right)$ for the other, and vice versa. Stimuli were chosen at $10 \mathrm{~dB}$ separations along an intensity continuum running from $100 \mathrm{~dB}$ to $60 \mathrm{~dB}$. For the counterpart pair trained at the high intensity end, a large difference in the speed of acquisition of differential responding and the terminal level of performance was obtained. This difference was attributable to a higher $S^{\Delta}$ rate for the group which had the more intense stimulus as $\mathrm{S}^{\Delta}$. The same effect appeared for some of the other groups, but it was graded as a function of the position of the stimuli on the continuum, so that at the lowest intensities, no measurable difference between the counterpart pair was found.

The present study was an attempt to replicate these findings using $5 \mathrm{~dB} \mathrm{~s}_{-} \mathrm{S}^{\Delta}$ differences. Only the effects at the most intense and least intense ends of the continuum were studied.

Method

Ss were 16 Sprague-Dawley derived, male, albino rats between $100-110$ days old at the start of the experiment. They were maintained at $80 \%$ of their freefeeding weights. Sound shielded enclosures equipped with speaker, retractable bar, water bottle, pellet dispenser, and food tray were used. The programming of auditory stimuli, reinforcements, and the recording of responses was automated and controlled from an adjacent room. The stimuli employed were $4 \mathrm{kHz}$ tones and are specified in terms of decibels attenuation from a $100 \mathrm{~dB}$ (re 0.0002 dynes $/ \mathrm{cm}^{2}$ ) reference tone. Therefore, the smaller the number which designates a given stimulus, the less the attenuation, and the greater the intensity. A more detailed description of the apparatus is given in Pierrel \& Sherman (1960).

There was an initial day of bar-training in which 100 reinforcements were received on an FI $10 \mathrm{sec}$. schedule, followed by 2 days $(2 \mathrm{hr} . /$ day) of training on the VI $1 \mathrm{~min}$. schedule which was to be in effect during subsequent $S^{D}$ presentations. During these sessions the stimulus which was to be $\mathrm{S}^{\mathrm{D}}$ for a given group was present at all times. Daily 4-hr. sessions of discrimination training were begun on the following day. The four groups of four rats each were trained as two counterpart pairs. For the high intensity pair, $0 \mathrm{~dB}$ and $5 \mathrm{~dB}$ were the stimuli, and for the low intensity pair, $35 \mathrm{~dB}$ and $40 \mathrm{~dB}$ were used. Each group is labelled according to its stimuli with $\mathrm{SD}_{\mathrm{as}}$ the

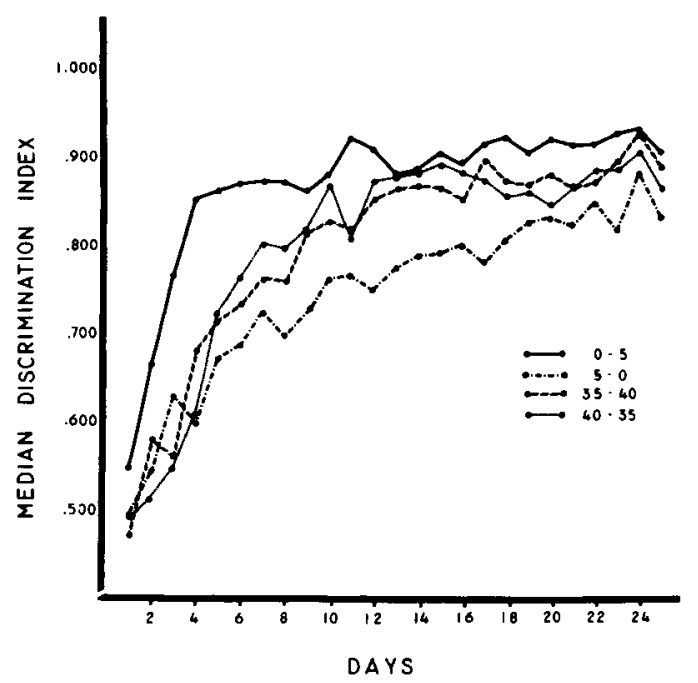

Fig. 1. Median Discrimination Indices for each group as a function of days during discrimination acouisition. 
first named. For example, the "0-5" group had $0 \mathrm{~dB}$ as $\mathrm{S}^{\mathrm{D}}$ and $5 \mathrm{~dB}$ as $\mathrm{S}^{\Delta}$. Discrimination training was run for 25 consecutive days, and the stimuli were presented according to a multiple VI 1 extinction schedule. During each hour, $15 \mathrm{~min}$. were devoted to $\mathrm{S}^{\mathrm{D}}$ presentations of $20 \mathrm{sec}$. to $3 \mathrm{~min}$. in duration, and $45 \mathrm{~min}$. to $\mathrm{S}^{\Delta}$ presentations of $40 \mathrm{sec}$. to $9 \mathrm{~min}$. in duration. The stimuli alternated according to a Gellerman series.

\section{Results and Discussion}

A Discrimination Index (D. I.) for each session was computed by dividing the number of $\mathrm{S}^{\mathrm{D}}$ responses by the total response output $\left(\mathrm{S}^{\mathrm{D}} / \mathrm{SD}+\mathrm{S}^{\Delta}\right) . \mathrm{S}^{-}$responses were multiplied by .33 to correct for the disproportionate amount of time devoted to this stimulus. The D. I. functions for all groups appear in Fig. 1. A large difference appears between the rate of acquisition and terminal level of performance for Groups $0-5$ and 5-0. The best performance of the four groups is that of the former, and the worst, that of the latter. The two low intensity groups (3540 and 40-35) fall in between and display no systematic differences. These results replicate those found by Sadowsky (1966) using counterpart pairs trained on $0-10$ vs. $10-0$ and $30-40$ vs. $40-30$. There is a difference, however, in the magnitude of the effect. Mean terminal performance D. I.s for Sadowsky's 0-10 group were about .972 and for the 10-0 group about .931, or a difference of .041 D. I. points. For the present study, mean terminal D. I.s for the 0-5 group were about .919 and for 5-0 about .846, or a difference of .073 D. I. points. Apparently, Stimulus Intensity Dynamism, when it appears at the higher in-

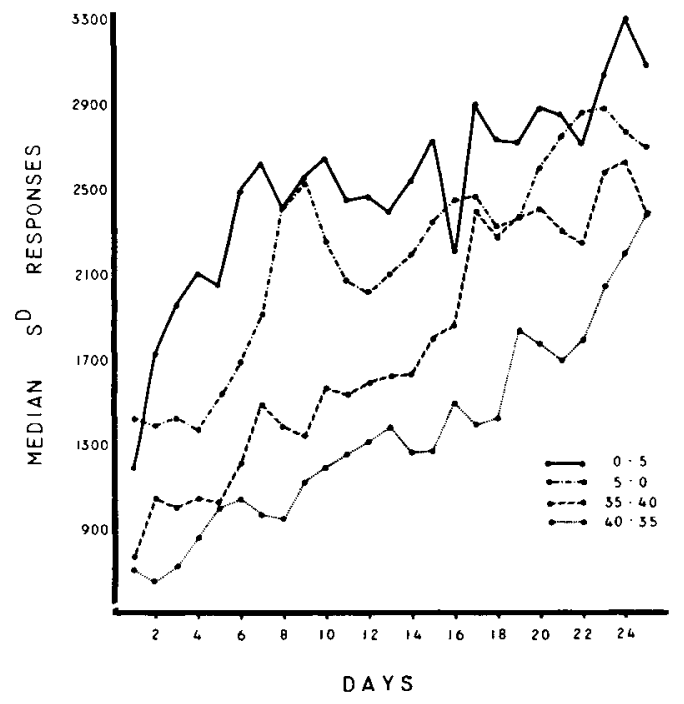

Fig. 2. Median total $\mathbf{S}^{\mathbf{D}}$ responses per session for each group in Fig. 1.

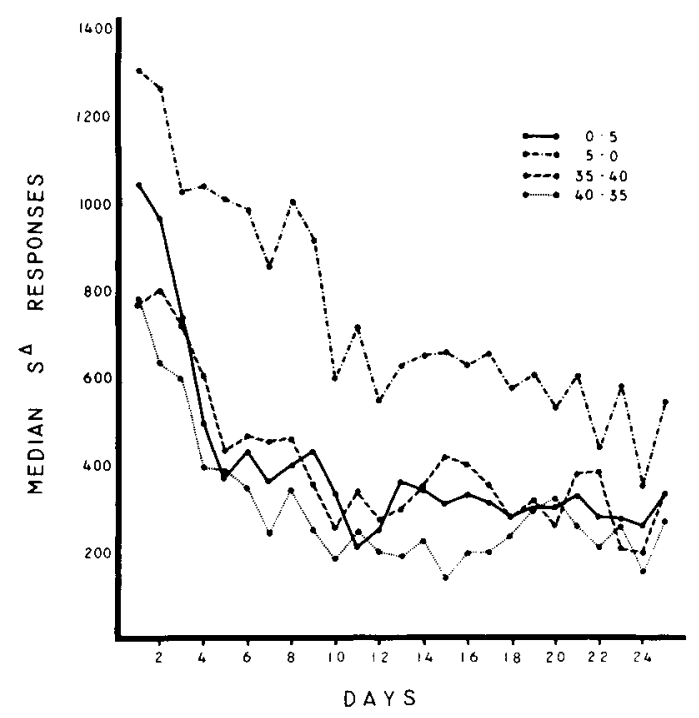

Fig. 3. Median total $\mathrm{s}^{\Delta}$ responses per session for each group in Fig. 1 .

tensities, is an inverse function of $\mathrm{S}^{\mathrm{D}}-\mathrm{S}^{\Delta}$ difference.

Another distinction between Sadowsky (1966) and the present study can be seen in the curves in Figs. 2 and 3 which plot $\mathrm{S}^{\mathrm{D}}$ and $\mathrm{S}^{\Delta}$ responding, respectively, over days. Sadowsky found no systematic differences in $S^{D}$ rates between his groups, but Fig. 2 clearly shows that rate of response is an increasing function of $\mathrm{S}^{\mathrm{D}}$ intensity (" $\mathrm{SD}$ Dynamism"). The curves in Fig. 3 show a consistent rate difference as a function of $\mathrm{s}^{\Delta}$ intensity (" $\mathrm{S}^{\Delta}$ Dynamism") only for the comparison between the highest intensity $S^{\Delta}(0 \mathrm{~dB})$ and the other three intensities. A more systematic investigation of the interaction of $\mathrm{S}^{\mathrm{D}}-\mathrm{S}^{\Delta}$ difference and $\mathrm{S}^{\mathrm{D}}$ and $\mathrm{S}^{\Delta}$ Dynamism is now underway in our laboratory.

\section{References}

Gray, J. A. Stimulus intensity dynamism. Psychol. Bull., 1965, 63, $180-196$

Hull, C. L. Stimulus intensity dynamism (V) and stimulus generalization. Psychol. Rev., 1949, 56, 67-76.

Pierrel, R., \& Sherman, J. G. Generalization of auditory intensity following discrimination training. J. exp. Anal. Behav., 1960. 3, 313-322.

Sadowsky, S. Discrimination learning as a function of stimulus location along an auditory intensity continuum. J. exp. Anal. Behav., 1966, 9, 219-225.

\section{Note}

1. This paper is based on part of a thesis submitted to the Graduate School of Brown University in partial fulfillment of the requirements for the degree of Master of Arts in the Department of Psychology. The research was conducted while the author was a PHS predoctoral fellow and was supported by NIH Grant HD-00928, Dr. Rosemary Pierrel, principal investigator. 